\title{
PROPAGAÇÃO DE Sebastiania schottiana MÜLL. ARG PELO MÉTODO DE ESTAQUIA EM DIFERENTES SUBSTRATOS
}

\author{
Jéssica Thalheimer de Aguiar ${ }^{1}$, Luis Paulo Baldissera Schorr², Tarik Cuchi ${ }^{3}$, Ana Cláudia da Silveira ${ }^{3}$, Monique
} Bohora Schlickmann ${ }^{3}$

\author{
'Doutoranda em Ciências Florestais - Universidade Estadual do Centro-Oeste, Irati, Paraná \\ ${ }^{2}$ Mestrando em Engenharia Florestal - Universidade do Estado de Santa Catarina, Lages, Santa Catarina \\ ${ }^{3}$ Mestre em Engenharia Florestal - Universidade do Estado de Santa Catarina, Lages, Santa Catarina \\ *Autor para correspondência: Jéssica Thalheimer de Aguiar, jeh.aguiar93@hotmail.com
}

\begin{abstract}
RESUMO: A espécie Sebastiania schottiana Müll. Arg, popularmente conhecida como Sarandi, possui dificuldade para a realização de sua reprodução sexuada, desta forma, a estaquia é um método alternativo utilizado na produção de mudas desta espécie. Neste sentido, o presente trabalho teve como objetivo avaliar a eficiência da propagação vegetativa por estaquia da espécie $S$. schottiana. Testou-se a sobrevivência e enraizamento de estacas, a partir de ramos adultos, que foram confeccionadas com cerca de $7 \mathrm{~cm}$ de comprimento, utilizando uma concentração de ácido indolbutírico (AIB) de $2000 \mathrm{mg} / \mathrm{L}$. 0 estaqueamento foi feito em tubetes de polietileno $(13,2 \mathrm{~cm}$ de altura e $5,2 \mathrm{~cm}$ de diâmetro) preenchidos com diferentes substratos em que se subdividiu os tratamentos, sendo eles T1: solo, T2: solo e AIB, T3: areia e AIB e o T4: vermiculita e AIB. Os parâmetros utilizados para avaliação após 45 dias de permanência em casa de vegetação foram: porcentagem de sobrevivência, porcentagem de estacas com brotações e a porcentagem de estacas enraizadas. Os dados foram submetidos ao teste de homogeneidade de variâncias, sendo as médias comparadas pelo teste não paramétrico de Kruskal-Wallis ao nível de $5 \%$ de probabilidade. Foi observado que o tratamento T3 (com substrato areia mais AIB) e T2 (com solo mais AIB), obtiveram maior destaque em comparação aos demais favorecendo a propagação vegetativa de $S$. schottiana.
\end{abstract}

PALAVRAS-CHAVE: enraizamento, estacas, propagação vegetativa.

\section{PROPAGATION OF Sebastiania schottiana MÜLL. ARG BY THE STACKING METHOD IN DIFFERENT SUBSTRATES}

\begin{abstract}
The study aimed to evaluate the efficiency of vegetative propagation by cuttings of the forest species Sebastiania schottiana Müll. Arg, popularly known as sarandi. We tested the survival and cuttings from adult branches, which were prepared with about $7 \mathrm{~cm}$, using an concentration of IBA (IBA) $2000 \mathrm{mg} / \mathrm{L}$. The staking was done in polyethylene tubes (13.2 cm in height and $5.2 \mathrm{~cm}$ in diameter) filled with different substrates in which the treatments were subdivided, being T1: soil, T2: soil and AIB, T3: sand and AIB and, finally, T4: vermiculite and AIB. The parameters used for evaluation after 45 days of stay in the greenhouse were: percentage of live cuttings, percentage of stakes with sprouting, and the percentage of rooting cuttings. The data were submitted to test homogeneity of variance and transformed into statistical analysis using the Assistat program and means were compared by Kruskal-Wallis test at $5 \%$ probability of error. It was observed that the treatment $\mathrm{T} 3$ (with substrate sand plus IBA) and T2 (with soil plus IBA), were more prominent in comparison to the others favoring the vegetative propagation of $S$. schottiana.
\end{abstract}

KEYWORDS: Rooting, piles, vegetative propagation.

\section{INTRODUÇÃO}

Pertencente à família Euphorbiacea, Sebastiania schottiana Müll. Arg, popularmente conhecida como Sarandi, é uma espécie arbórea em que a estaquia é considerada um método alternativo utilizado para sua produção de mudas, visto que a mesma possui dificuldade para a realização de sua reprodução sexuada (Deschamps \& Pinto, 1995). 
Desse modo, torna-se necessário para a espécie, a utilização da técnica de produção de mudas por estaquia, por proporcionar mudas que mantenham as características genotípicas da planta, em curto período de tempo, e no caso da espécie em estudo, a multiplicação realizada por essa técnica auxiliará na manutenção da estabilidade ecológica ambiental, uma vez que irá proporcionar uma melhor produção de mudas da espécie, aumentando a disponibilidade de mudas de qualidade a serem utilizadas para esse fim.

Existem vários métodos para a propagação vegetativa de plantas, sendo um deles a estaquia. Estaquia é o termo utilizado para designar o método de propagação no qual um segmento da planta matriz é retirado, colocado em condições ambientais favoráveis e induzido a formar raízes e brotos, obtendo-se uma nova planta (Paulino et al., 2012).

Vários fatores devem ser levados em consideração para a propagação das espécies por estaquia, sendo que podemos destacar as características da espécie que se deseja propagar, o tipo de substrato e as auxinas utilizadas (Silva et al., 2011).

O substrato tem a função de dar a sustentação e nutrição para a planta, sendo que devem ser consideradas suas características físico-químicas, pois as mesmas influenciam na formação e desenvolvimento do sistema radicular e no estado nutricional das plantas (Moraes et al., 2012; Dalanhol et al., 2016).

Dentre as auxinas, destaca-se 0 ácido indolbutírico (AIB), o qual é uma das auxinas mais utilizadas, devido à sua alta atividade de morfogênese, ocasionando a aceleração do enraizamento, sendo menos fotolábil e fitotóxica que outras auxinas sintéticas (Moura et al., 2011). Esse fato pode ser explicado devido à capacidade que esses reguladores vegetais têm em modificar processos fisiológicos e morfológicos das espécies (Vilaninho \& Cândido, 2014).

Diante do exposto, objetivou-se avaliar o efeito de diferentes substratos na propagação vegetativa, pelo método de estaquia, de Sarandi (S. schottiana).

\section{MATERIAL E MÉTODOS}

Este experimento utilizou estacas provenientes de diferentes árvores matrizes de um fragmento florestal localizado no município de Frederico Westphalen/RS, coordenadas $27^{\circ} 22^{\prime \prime}$; $53^{\circ} 25^{\prime \prime} \mathrm{W}$, a $500 \mathrm{~m}$ de altitude, e foram confeccionadas com aproximadamente $7 \mathrm{~cm}$ de comprimento. Após este processo, as bases das estacas foram embebidas em solução de AIB 2000 mg/L e estaqueadas em tubetes de polietileno de 215,64 $\mathrm{cm}^{3}$ de volume com diferentes substratos, em que se subdividiu os tratamentos, sendo estes apresentados na Tabela 1.

Tabela 1. Tratamentos utilizados para propagação vegetativa de Sebastiania schottiana Müll. Arg

\begin{tabular}{ccc}
\hline TRATAMENTO & SUBSTRATO & REGULADOR DE CRESCIMENTO \\
\hline T1 & Solo & - \\
T2 & Solo & AIB $2000 \mathrm{mg} / \mathrm{L}$ \\
T3 & Areia & AIB $2000 \mathrm{mg} / \mathrm{L}$ \\
T4 & Vermiculita & AIB $2000 \mathrm{mg} / \mathrm{L}$ \\
\hline
\end{tabular}

0 delineamento experimental foi inteiramente casualizado. Avaliaram-se quatro tratamentos, usando quatro repetições e cada unidade experimental foi formada por oito estacas.

0 enraizamento das estacas foi avaliado após 45 dias de permanência em casa de vegetação com irrigação controlada pelo método de aspersão, com turnos de regas de três vezes ao dia, com uma vazão média de $7 \mathrm{~mm}$ diário. As variáveis avaliadas foram a porcentagem de sobrevivência, a porcentagem de estacas com brotações e a porcentagem de estacas enraizadas.

Como os dados não atenderam ao pressuposto de normalidade usou-se estatística não-paramétrica (Teste de Kruskal-Wallis a $5 \%$ de probabilidade de erro).

\section{RESULTADOS E DISCUSSÃO}

Os resultados estatísticos para as variáveis analisadas na propagação vegetativa de S. schottiana são apresentados na Tabela 2. 
Tabela 2. Resultados estatísticos para as variáveis analisadas na propagação vegetativa de Sebastiania schottiana Müll. Arg

\begin{tabular}{cccc}
\hline \multirow{2}{*}{ TRATAMENTO } & \multicolumn{3}{c}{ VARIÁVEIS ANALISADAS } \\
\cline { 2 - 4 } & Sobrevivência $(\%)$ & Brotações (\%) & Estacas Enraizadas (\%) \\
\hline T1 & $25 \mathrm{~b}$ & $21,88 \mathrm{~b}$ & $15,63 \mathrm{~b}$ \\
T2 & $40,63 \mathrm{ab}$ & $37,5 \mathrm{a}$ & $34,38 \mathrm{a}$ \\
T3 & $46,88 \mathrm{a}$ & $31,25 \mathrm{ab}$ & $28,13 \mathrm{ab}$ \\
T4 & $28,13 \mathrm{ab}$ & $25 \mathrm{ab}$ & $25 \mathrm{ab}$ \\
\hline
\end{tabular}

Em que: T1: Solo, T2: solo e AIB, T3: areia e AIB e o T4: vermiculita e AIB. Médias seguidas de mesma letra não diferem estatisticamente pelo Teste de Kruskal-Wallis a 5\% de probabilidade de erro.

A partir dos resultados obtidos, observou-se uma baixa taxa de sobrevivência das estacas, onde se evidenciou que nenhum dos tratamentos proporcionou uma taxa de sobrevivência superior a $50 \%$.

A maior taxa de porcentagem de sobrevivência foi obtida para o tratamento T3 (areia e AIB a 2000 $\mathrm{mg} / \mathrm{L}$ ) apresentando uma média de $46,88 \%$ de estacas vivas. Entretanto, o mesmo não diferiu estatisticamente do tratamento T2 (solo e AIB a $2000 \mathrm{mg} / \mathrm{L}$ ) e T4 (vermiculita e AIB a $2000 \mathrm{mg} / \mathrm{L}$ ). Contudo, T3 foi 0 único tratamento que se diferenciou de $\mathrm{T} 1$ (solo sem presença de AIB), obtendo-se um acréscimo de 46,7\% no número de estacas vivas em relação ao tratamento sem indutor de enraizamento.

Em estudo semelhante realizado por Cardoso et al. (2011) também foi verificado que não houve interação entre as concentrações de AIB e diferentes substratos, de acordo com a porcentagem de sobrevivência, não sendo observadas diferenças significativas entre esses fatores.

No caso da não diferenciação dos resultados, esta pode estar ligada com a alta capacidade que a espécie tem em vigorar, fato que corrobora com Yamanoto et al. (2013), que no estudo da estaquia de amora-preta xavante (Rubus spp. L.), os diferentes substratos utilizados não influenciaram significativamente na porcentagem de sobrevivência das estacas. Destacando que tanto a Rubus spp. quanto a $S$. schottiana apresentam baixa capacidade de vigor.

Dentre os tratamentos em que ocorreram brotações, a maior porcentagem média foi obtida no tratamento T2 que teve em média $37,5 \%$ de estacas com brotações, seguido pelo T3 com 31,25\% e T4 com $25 \%$. O T1 apresentou menor taxa de brotações, com apenas $21,88 \%$ das estacas apresentando brotos.
A análise estatística constatou que em relação às brotações das estacas houve diferenciação significativa entre os tratamentos, ou seja, os tratamentos analisados promoveram diferentes taxas de brotação, sendo que T1 não se diferiu estatisticamente do T3 e T4, no entanto, diferiu do T2, que contém a maior média de brotações.

Evidenciando que para melhores resultados de brotação de estacas de $S$. schottiana é necessário a utilização de AIB. Singh et al. (2011) em estudo de estaquia de Bougainvillea glabra Choisy com a utilização de AIB, também verificaram maiores porcentagens de brotação nas estacas lenhosas tratadas com $2000 \mathrm{mg} / \mathrm{L}$ de AlB. Costa et al. (2015) também encontraram maior porcentagem de estacas de Bougainvillea spectabilis L. com brotos, utilizando AIB em concentração de 2000 mg/L.

Os resultados obtidos em relação à brotação das estacas podem estar relacionados com o processo biológico envolvido na emissão de brotos, pois nesse processo a energia é originária da respiração, ou seja, moléculas de 02 são necessárias para "queimar" essas substâncias (Manhães et al., 2015). Desta forma, dentre um período de cerca de 60 dias as reservas das estacas são utilizadas para que ocorra à emissão dos brotos, e se reduzido essa dependência na medida em que o sistema radicular se desenvolve é aumentando a superfície ativa de absorção de água e nutrientes do solo (Magro et al., 2011).

Quanto a variável enraizamento podemos observar que houve resultados significativos, e que o tratamento que alcançou uma maior porcentagem foi o T2 que teve uma média de $34,38 \%$ de estacas enraizadas. T1 não se diferiu estatisticamente do T3 e T4, no entanto, diferiu do T2, que contém a maior média de enraizamento. 
No presente estudo foram encontradas taxas superiores quando comparadas ao trabalho de Santos et al. (2011) que analisando estacas lenhosas de $S$. schotthiana e utilizando diferentes dosagens de AIB, alcançaram uma taxa de enraizamento igual a $6 \%$. Tal fator pode ser explicado pela utilização de apenas um substrato composto por vermiculita expandida e casca de arroz carbonizada na proporção volumétrica de 1:1.

Segundo Oliveira et al. (2015) o estímulo ao enraizamento diverge entre espécies pela concentração de auxina existente nos tecidos vegetais. $\mathrm{O}$ autor ainda relata que uma fonte externa de auxina pode gerar aumento de sua concentração, e consequentemente, induzindo à formação do sistema radicular.

Assim, como visto por Moura et al. (2015) a utilização de auxinas favoreceu o enraizamento, sendo que a testemunha se mostrou com uma quantia relativamente inferior de estacas com raízes, o que se assemelha com o que foi encontrado nesta pesquisa.

Contudo, devem ser realizados estudos complementares que possibilitem maior conhecimento sobre a espécie, época de colheita, tempo de enraizamento, aplicação de auxinas em maiores concentrações, bem como tipo e proporção de substratos.

A aplicação de AIB na dosagem de 2000 $\mathrm{mg} / \mathrm{L}$, favoreceu todos os parâmetros analisados (sobrevivência, brotações e enraizamento), de forma considerável em relação à testemunha. 0 tratamento que obteve melhores resultados foi o (T3) com substrato contendo areia.

Sugere-se a realização de novos estudos com diferentes substratos e dosagens de AIB ou outras auxinas, para que se obtenha a melhor forma de enraizamento da espécie em estudo.

\section{REFERÊNCIAS BIBLIOGRÁFICAS}

Caldeira, M.V.W.; Peroni, L.; Gomes, D.R.; Delarmelina, W.M.; Trazzi, A. Diferentes proporções de biossólido na composição de substratos para a produção de mudas de timbó (Ateleia glazioveana Baill). Scientia Forestalis, 2012, 40, 15-22.

Cardoso, C.; Yamamoto, L.Y; Preti, E.A.; Assis, A.M.; Neves, C.S.V.J.; Roberto, S.R. AIB e substratos no enraizamento de estacas de pessegueiro 'Okinawa' coletadas no outono.Semina: Ciências Agrárias, 2011, $32,1307-1314$

Costa, E.M.; Loss, A.; Pereira, H.P.N.; Almeida, J.F. Enraizamento de estacas de Bougainvillea spectabilis Willd. com 0 uso de ácido indolbutírico. Acta Agronômica. 2015, 64, 221-226.

Dalanhol, S.J.; Nogueira, A.C.; Gaiad, S.; Kratz, D. Efeito de fungos micorrízicos arbusculares e da adubação no crescimento de mudas de Eugenia uniflora L., produzidas em diferentes substratos. Revista Brasileira de Fruticultura, 2016, 38, 117-128.

Deschamps, C.; Pinto J.E.B.P. Enraizamento in vitro de microestacas e micropropagação de gemas axilares de sarandi (Sebastiania schottiana Müell. Arg.). Ciência Rural, 1995, 25, 389-393.

Frassetto, E.G.; Franco, E.T.H.; Kielse, P.; Amaral, V.F.M. Enraizamento de estacas de Sebastiania schottiana Müll. Arg. Ciência Rural, 2010, 40, 25052509.

Hoffmann A. Fruticultura comercial: propagação de plantas frutíferas. Lavras: UFLA/FAEPE, 1996, 319p.

Lima J.D; Bolfarini, A.C.B.; Modenese-Gorda, S.H.; Moraes, W.S. Propagação de Camellia sinensis: efeito do genótipo, estaca, substrato, recipiente e ácido indolbutírico. Horticultura Brasileira, 2013, 31, 74-79.

Magro, F.J.; Takao, G.; Camargo, P.E.; Takamatsu, S.Y. Biometria em cana-de-açúcar. LPV0684: Produção de Cana-de-Açúcar, USP, Escola Superior de Agricultura "Luiz de Queiroz", Piracicaba, SP, 2011.

Manhães, C.M.C.; Garcia, R.F.; Francelino, F.M.A.; Francelinho, H.O.; Coelho, C. Fatores que afetam a brotação e o perfilhamento da cana-de-açúcar. Vértices, 2015, 17, 163-181.

Moraes, F.A.; Góes, G.B.de.; Costa,M.E.da.; Melo, I.G.C.; Veras, A.R R.;. Cunha, G.O.deM. Fontes e proporções de esterco na composição de substratos para produção de mudas de jaqueira. Revista Brasileira de Ciências Agrárias - Brazilian Journal Of Agricultural Sciences, 2012, 7, 784-789. 
Moura, A.P.C; Salla, V.P.; Lima, D.M. Enraizamento de estacas de bougainvillea com concentrações de ácido naftaleno acético. Scientia Agraria, 2015, 16, 57-61.

Moura, A.P.; Campos, R.A.S.; Seabra, J.S.; Lima, G.P.P. Enraizamento de estacas de bertalha em diferentes concentrações de IBA. Horticultura Brasileira, 2011, 29, 1728-1734.

Oinam, G. Adventitious Root formation in ornamental plants: I. general overview and recent successes. Propagation of Ornamental Plants, 2011, 11, 78-90.

Oliveira, J.A.R.; Koefender, J.; Manfio, C.E.; Golle, D. P. Reis, J.D. Tipos de estacas e uso de AIB na propagação vegetativa de fisális. Revista Agro@mbiente On-line, 2015, 9, 342-346.

Paulino, R.C.; Santos L.W.; Coelho, M.F.B. Propagação por estaquia de Croton zehntneri Pax et Hoffm. (Euphorbiaceae) em diferentes concentrações de indutores de enraizamento. Revista Verde, 2012, 7, 2933.

Santos, J.P.; Davide, A.C.; Teixeira, F.L.A; Melo, A.J.S.; Melo, L.A. Enraizamento de estacas lenhosas de espécies florestais. Cerne, 2011, 17, 293-301.

Silva, S.R.; Rodrigues, K.F.D.; Filho J.A.S. Propagação de Árvores Frutiferas. Piracicaba: USP/ESALQ/Casa do produtor rural, 63p, 2011.

Singh, K.K., Rawat, J.M.S.; Tomar, Y. K. Influence of IBA on rooting potential of torch glory Bougainvillea glabra during winter season. Journal of Horticultural Science \& Ornamental Plants, 2011, 3,162-165.

Yamamoto, L.Y.;, Koyama, R.; Assis, A.M.; Borges, W.F.S.; Oliveira, I.R.; Roberto, S.R. Substratos no enraizamento de estacas herbáceas de amora-preta Xavante. Revista Ciência Rural, 2013, 43, 15-20. 